\title{
Respiratory Virus Infections in People Over 14 Years of Age in Poland in the Epidemic Season of 2017/18
}

\author{
K. Szymański, K. Łuniewska, E. Hallmann-Szelińska, \\ D. Kowalczyk, R. Sałamatin, A. Masny, and L. B. Brydak
}

\begin{abstract}
People most at risk of influenza complications are the elderly with impaired immunity. Clinical picture of influenza virus infection includes symptoms such as chills, increased body temperature, dry cough, chest pain, or dizziness as well as headaches and muscle aches. In the diagnosis of influenza, quick and effective tests are necessary. Sensitive diagnostic methods of molecular biology require more time, but the result firmly confirm or exclude the presence of the genetic material of influenza or other respiratory viruses. Influenza vaccination plays an important role in combating influenza infection. Unfortunately, the awareness of vaccination benefits is insufficient in Poland. In this study we demonstrate the results of examination of 4,507 people aged over 14 years toward the influenza infection in the epidemic season of 2017/18. Most of the confirmed infections were reported in older people aged over 65 , a high-risk population group. A low percentage of the vaccinated
\end{abstract}

K. Szymański ( $\bowtie)$, K. Łuniewska, E. HallmannSzelińska, D. Kowalczyk, A. Masny, and L. B. Brydak Department of Influenza Research, National Influenza Center, National Institute of Public Health - National Institute of Hygiene, Warsaw, Poland e-mail: kszymanski@pzh.gov.pl

R. Sałamatin

Department of General Biology and Parasitology, Medical University of Warsaw, Warsaw, Poland population may affect an increased number of confirmed influenza viruses in the elderly. The findings demonstrate a need to increase awareness of vaccination benefits, which is particularly essential to avoid influenza infection in the elderly.

\section{Keywords}

IImmunity · Elderly · Epidemic season ·

Influenza $\cdot$ Respiratory infection $\cdot$ Respiratory virus vaccination

\section{Introduction}

There are four types of influenza viruses: A, B, C, and D. Types A and B are responsible for seasonal outbreaks. Type A viruses are divided into subtypes, and currently, there are $\mathrm{A} / \mathrm{H} 1 \mathrm{~N} 1 /$ pdm09 and $\mathrm{A} / \mathrm{H} 3 \mathrm{~N} 2 /$ subtypes circulating in the population, while influenza $\mathrm{B}$ viruses belong to two lineages: B/Victoria and B/Yamagata. At present, only type A viruses are responsible for causing a pandemic flu (CDC 2018a; Ghebrehewet et al. 2016). The population groups most vulnerable to complications associated with influenza virus infections are the elderly, children under 5 years of age, people with chronic diseases, pregnant women, health care workers, and people with immunological diseases that impair natural immunity (WHO 2018). 
General symptoms of influenza virus infection consist of a sudden appearance of high fever, cough, headache, myalgia, and general malaise. Respiratory symptoms include sore throat and dry painful cough. In addition, symptoms from other organ systems may appear such as anorexia, dizziness, muscle pain, and nausea and vomiting in children. These symptoms would appear after a short incubation period of 1-2 days (ECDC 2018; Paules and Subbarao 2017).

An effective laboratory diagnosis of influenza is necessary to promptly provide appropriate antiviral treatment of infection. The fastest diagnostic method is a rapid influenza swab-based testing that shows sensitivity of about $70 \%$ or between $27 \%$ and $61 \%$, depending on the source. The advantage of this test the speed as it takes 15-30 $\mathrm{min}$ to complete and the ease of performing it as it can be done outside a specialized laboratory. The disadvantage, however, is a substantial risk of false negative results due to rather low sensitivity or false positive results due to misinterpretation of weak bands in the test (Dwyer et al. 2006). The reverse transcription-polymerase chain reaction (RT-PCR) remains the most sensitive method for the detection of viral genetic material. This method takes less than a working day to complete a test and the advantage is the ability to detect more than just one respiratory virus when a multiplex set is used. Another possible method is the immunofluorescence-based reaction of hemagglutination inhibition, which defines the antiviral antibody titers (Kissova et al. 2014).

Protective vaccination is important in combating the influenza virus. Studies have shown that people vaccinated against influenza have significantly fewer influenza-like virus infections (Taksler et al. 2015). The knowledge about the benefits of influenza vaccination is insufficient in Poland, among both the medical personnel and the lay public. Parents do not vaccinate their children against influenza due mostly to the lack of knowledge about the benefits of it (WozniakKosek et al. 2015). The risk-benefit ratio of vaccination has changed downward over the past decades, reducing mortality, particularly among adults (Pfleiderer 2014; Brydak 2012). A greater coverage rate of vaccination also substantially reduces the economic impact of influenza infection. A previous study showed that about $50 \%$ of older people who had been vaccinated developed influenza in the season of $2015 / 16$, but they did not require hospitalization, which points to a mild course of the infection. Influenza vaccine also is fully effective in older people with the underlying chronic pathological conditions (Rondy et al. 2017). The present study seeks to determine the incidence of influenza infection in persons aged over 14 years in the epidemic season of 2017/18 in Poland.

\section{Methods}

\subsection{Patients and Samples}

In this study we examined over 4507 specimens obtained from patients over 14 years of age during the epidemic influenza season of 2017/18 in Poland. The specimens consisted of throat and nasal swabs or of bronchial tree lavage. The patients were divided into the following age groups: $15-25,26-44,45-64$, and $65+$ years of age. Genetic material of respiratory viruses was isolated from the specimens, using $200 \mu \mathrm{L}$ samples suspended in sterile phosphate-buffered saline solution. Viral RNA was isolated with the Maxwell 16 Viral Total Nucleic Acid Purification Kit (Promega Corporation; Madison, WI). The procedure was carried out in accordance with the manufacturer's instructions.

\subsection{Molecular Procedures}

\section{Real-Time Polymerase Chain Reaction (PCR)}

The primers and probes were obtained from the International Reagent Resource run by the Centers for Disease Control and Prevention (CDC) in the US. Briefly, RNA was reversely transcribed at $50{ }^{\circ} \mathrm{C}$ for $30 \mathrm{~min}$. The DNA obtained was subjected to the initial denaturation process ( 1 cycle at $95{ }^{\circ} \mathrm{C}$ for $2 \mathrm{~min}$ ), followed by 45 cycles of amplification consisting of denaturation at $95^{\circ} \mathrm{C}$ for $15 \mathrm{~s}$, annealing at $55^{\circ} \mathrm{C}$ for $10 \mathrm{~s}$, 
and elongation at $72{ }^{\circ} \mathrm{C}$ for $20 \mathrm{~s}$. Positive control was the viral RNA obtained from the vaccine strains for the epidemic season of 2017/18 (A/Michigan/45/2015 pdm09-like virus, A/HongKong/4801/2014, and B/Brisbane/60/ 2008), and negative control was RNase-free water. The PCR reactions were performed using the LightCycler 2.0 System (Roche Diagnostics; Rotkreuz, Switzerland).

Conventional Multiplex PCR The tests were performed to identify the presence of the following respiratory viruses in the sampled material: influenza A and B; adenovirus; respiratory syncytial virus (RSV) A and $\mathrm{B}$; human metapneumovirus (hMPV); human coronavirus (hCoV); human parainfluenza viruses 1, 2, 3; human bocavirus; and enterovirus. The RV15 OneStep ACE Detection Kit (Seegene; Seoul, South Korea) was used to determine the infection by the influenza-like viruses above outlined.

\section{Results and Discussion}

Distribution of the investigated specimens by age of patients is presented in Fig. 1. The patients aged $65+$ constituted the most numerous group, followed by the groups of 45-64 and 26-44 years of age, with the least numerous being 15-25 years group. The prevailing type of infection was influenza B virus, followed by type A unsubtyped and the A/H1N1/pdm09 and A/H3N2/ subtypes (Fig. 2).

Concerning the prevailing influenza type B infections, the greatest incidence was reported in persons aged $65+$, followed by the groups of $15-25,45-64$, and 26-44 years of age. The majority of infections with type A virus unsubtyped were recorded in the group of 26-44 years of age, followed by the groups of 45-64 and 65+ years of age, with the least frequent in the 15-25 years group. The $\mathrm{A} / \mathrm{H} 1 \mathrm{~N} 1 / \mathrm{pdm} 09$ subtype was noted, in decreasing order of frequency, in the groups of 26-44, $45-64,15-25$, and $65+$ years of age. The prevalence of infections with the $\mathrm{A} / \mathrm{H} 3 \mathrm{~N} 2 /$ subtype did not exceed $1.5 \%$ in any of the age groups. This subtype was the most prevalent pathogen in the group of 15-25 years of age, with a decreasing incidence in the successive age groups to reach a barely noticeable level of $0.29 \%$ in the elderly (Fig. 3).

In contradistinction to influenza infections, influenza-like infections with respiratory viruses were most often diagnosed in the youngest adult group of 15-25 years of age. One half of these infections were due to RSV, followed by PIV-1 and $2, \mathrm{hCoV}$, and hMPV. In the group of 26-44 years of age, a similar number of respiratory viruses was detected, with RSV, PIV-2 and 3 , and rhinovirus being the most frequently
Fig. 1 Percentage distribution of investigated specimens by patients' age during the influenza epidemic season of 2017/18 in Poland

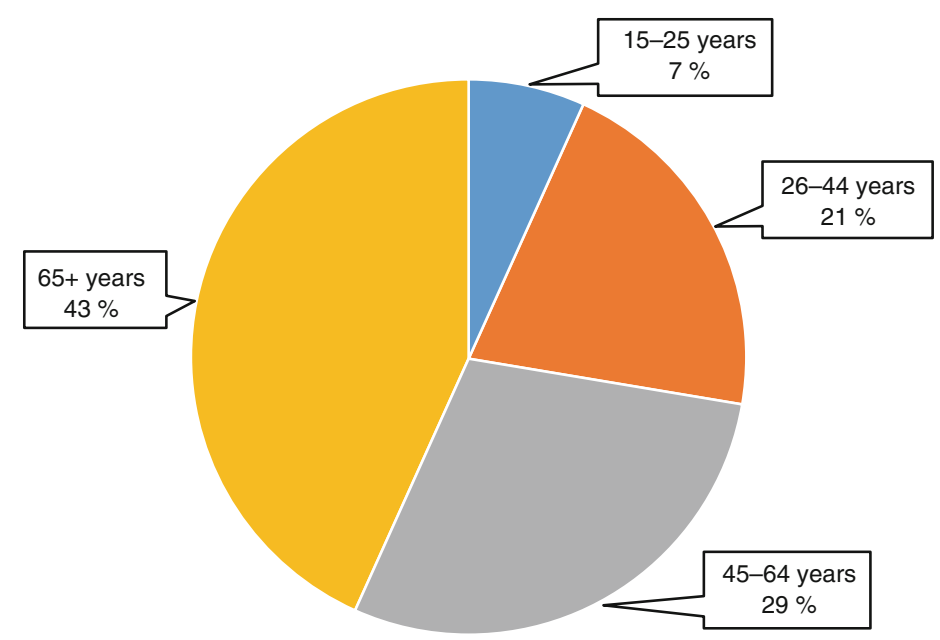


Fig. 2 Prevalence of confirmed infections with influenza viruses in patients over 14 years of age during the epidemic season of 2017/18 in Poland

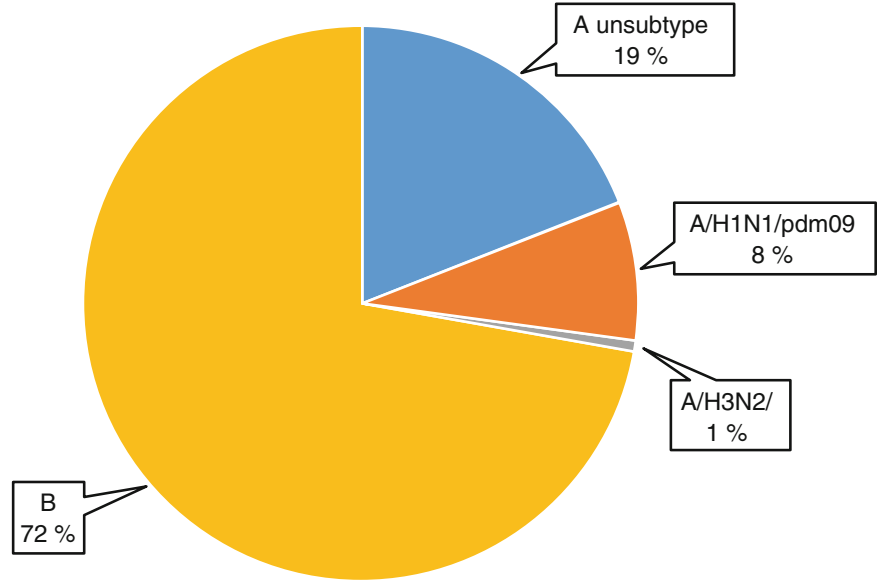

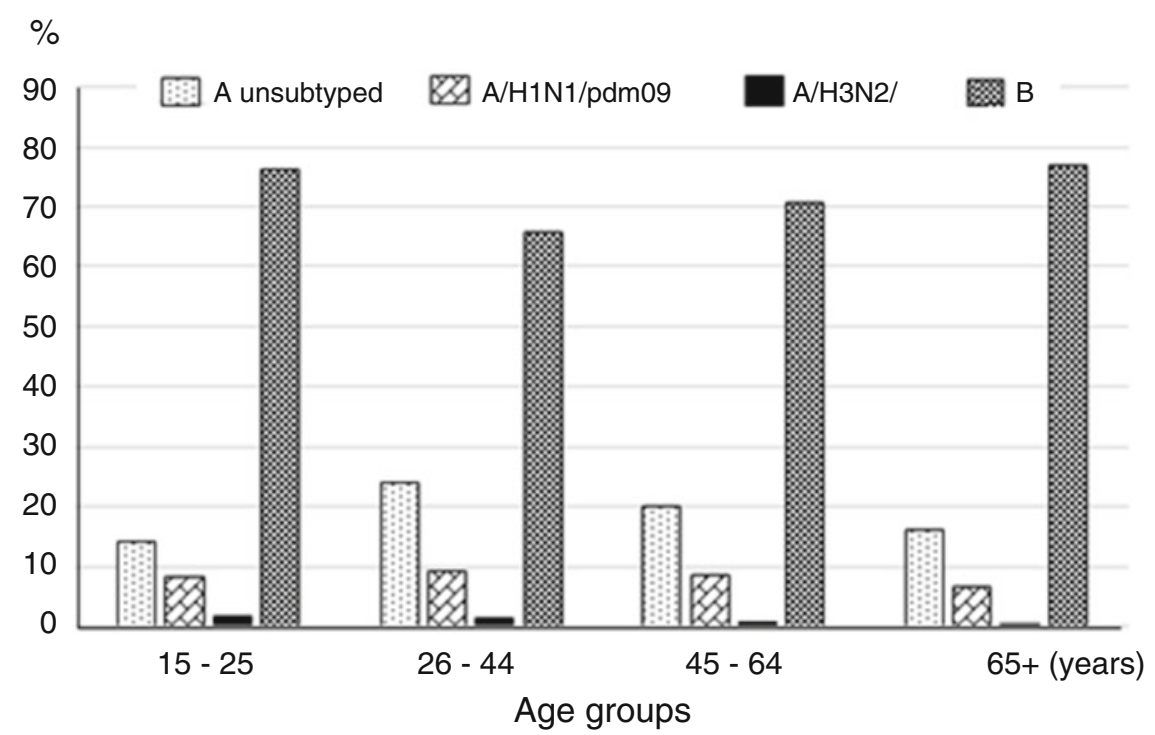

Fig. 3 Percentage distribution of confirmed infections with influenza viruses and their subtypes by successive age groups during the epidemic season of 2017/18 in Poland

detected. The frequency of detection of respiratory viruses decreased with patients' age. Fewer viruses were detected in the 45-64 age group, and they were identified as RSV, PIV-1, and coronavirus. The most seldom infections were reported in the elderly, in whom RSV was most frequently, followed by PIV-1 (Fig. 4).

In the epidemic season of $2017 / 18$ in Poland, there were 2,971,031 cases and suspected cases of infection with influenza viruses in persons over
14 years of age, of which 2,455,933 cases occurred in people aged 15-64 years and 515,098 in those over 65 years of age. The percentage of people vaccinated against influenza in the fall of 2017 was $1.57 \%$ and $7.52 \%$ in the respective cohorts. For comparison, the vaccination rate was $3.7 \%$ in the $2017 / 18$ season in the population of the entire country (PZH 2018). To protect the population from complications resulting from influenza infection, it is paramount 


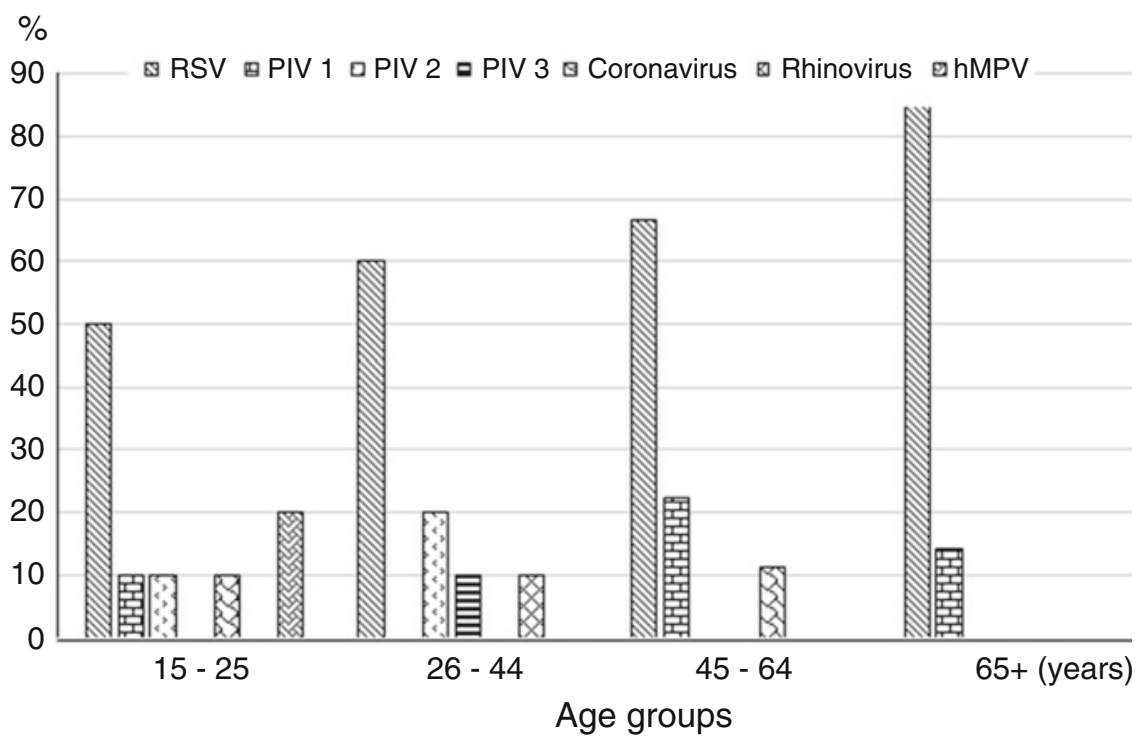

Fig. 4 Prevalence of confirmed infections with respiratory viruses in patients over 14 years of age during the epidemic season of 2017/18 in Poland. RSV respiratory syncytial virus, $P I V$ parainfluenza virus, $h M P V$ human metapneumovirus

to increase the vaccination coverage, mainly in people at risk of infection. The greater the number of people inoculated in their environment, the lower is the general risk of infection. Thus, those who cannot be vaccinated against influenza, for instance, due to allergies to vaccine ingredients, such as gelatin or some antibiotics, which often concerns children below the age of 6 months, would also be protected to an extent on the basis of collective resistance (CDC 2018b). Previous studies have shown that the vaccination coverage in a population should range from $33 \%$ to $73 \%$ to maximize the benefits of collective immunity and to reduce the transmission of influenza viruses (Logan et al. 2018; Plans-Rubió 2012). The present report demonstrates that influenza viruses can infect an adult person of any age. A study by Caini et al. (2018) have pointed, however, to the possibility of some age-dependent patterns of infection in that influenza $\mathrm{B}$ is more frequently detected in older children, while the subtype $\mathrm{A}(\mathrm{H} 3 \mathrm{~N} 2)$ is more common in the elderly.

In the 2017/18 influenza epidemic season, a larger number of specimens taken from people above 14 years of age were analyzed compared to the past 2016/17 season; 4507 and 2882, respectively (Kowalczyk et al. 2018). The distribution of infections among the age groups was, however, similar in the two seasons, with the larger number of positive results recorded in people over 65 years of age and a decreasing number of infections with decreasing age, with the smallest number in the 15-25 years group. In conclusion, the findings of this report emphasize a need to increase awareness of vaccination benefits, which is particularly essential to avoid influenza infection in the elderly.

Acknowledgments Supported by NIP-NIH thematic subject 3/EM.1. The authors express thanks to the physicians and employees of VSESs in the SENTINEL program for their input in the influenza surveillance in Poland.

Conflicts of Interest The authors declare no conflict of interests in relation to this article.

Ethical Approval All procedures performed in studies involving human participants were in accordance with the ethical standards of the institutional and/or national research committee and with the 1964 Helsinki declaration and its later amendments or comparable ethical standards. The study was approved by an institutional Ethics Committee. 
Informed Consent Informed consent was obtained from all individual participants included in the study before collection of nasopharyngeal samples.

\section{References}

Brydak LB (2012) Influenza - an age old problem. Hygeia Public Health 47(1):1-7

Caini S, Spreeuwenberg P, Kusznierz GF et al (2018) Distribution of influenza virus types by age using case-based global surveillance data from twenty-nine countries, 1999-2014. BMC Infect Dis 18(1):269

CDC (2018a). https://www.cdc.gov/flu/about/viruses/ types.htm. Accessed on 4 Oct 2018

CDC (2018b). https://www.cdc.gov/flu/professionals/vac cination/vaccine_safety.htm. Accessed on 4 Oct 2018

Dwyer DE, Smith DW, Catton MG, Barr IG (2006) Laboratory diagnosis of human seasonal and pandemic influenza virus infection. Med J Aust 185(10 Suppl): S48-S53

ECDC (2018). https://ecdc.europa.eu/en/seasonal-influ enza/facts/factsheet. Accessed on 17 Aug 2018

Ghebrehewet S, MacPherson P, Ho A (2016) Influenza. BMJ 355:i6258

Kissova R, Svitok M, Klement C, Madarova L (2014) Factors affecting the success of influenza laboratory diagnosis. Cent Eur Public Health 22(3):164-169

Kowalczyk D, Szymański K, Cieślak K, HallmannSzelińska E, Brydak LB (2018) Respiratory infections with particular emphasis on influenza virus activity in persons over 14 years of age in the epidemic season 2016/2017 in Poland. Adv Exp Med Biol 1108:75-80
Logan J, Nederhoff D, Koch B, Griffith B, Wolfson J, Awan FA, Basta NE (2018) What have you HEARD about the HERD? Does education about local influenza vaccination coverage and herd immunity affect willingness to vaccinate? Vaccine 36(28):4118-4125

Paules C, Subbarao K (2017) Influenza. Lancet 390 (10095):697-708

Pfleiderer M, Trouvin JH, Brasseur D, Gränstrom M, Shivji R, Mura M, Cavaleri M (2014) Summary of knowledge gaps related to quality and efficacy of current influenza vaccines. Vaccine 32(35):4586-4591

Plans-Rubió P (2012) The vaccination coverage required to establish herd immunity against influenza viruses. Prev Med 55(1):72-77

PZH (2018). http://wwwold.pzh.gov.pl/oldpage/epimeld/ grypa/index.htm. Accessed on 4 Oct 2018

Rondy M, Larrauri A, Casado I et al (2017) 2015/16 seasonal vaccine effectiveness against hospitalisation with influenza $\mathrm{A}(\mathrm{H} 1 \mathrm{~N} 1)$ pdm09 and B among elderly people in Europe: results from the I-MOVE+ project. Euro Surveill 22(30):pii: 30580. https://doi.org/10. 2807/1560-7917.ES.2017.22.30.30580

Taksler GB, Rothberg MB, Cutler DM (2015) Association of influenza vaccination coverage in younger adults with influenza-related illness in the elderly. Clin Infect Dis 61(10):1495-1503

WHO (2018). http://www.who.int/en/news-room/factsheets/detail/influenza-(seasonal). Accessed on 17 Aug 2018

Wozniak-Kosek A, Mendrycka M, Saracen A, Kosek J, Hallmann-Szelińska E, Zielnik-Jurkiewicz B, Kempińska-Mirosławska B (2015) Vaccination status and perception of influenza vaccination in the polish population. Adv Exp Med Biol 836:41-46 